\title{
Application of Double Strength Reduction Factor Method in the Stability Analysis of Rock Slopes
}

\author{
Jiawei Lu $\mathbb{D}^{1},{ }^{1}$ Jixun Zhang $\mathbb{D}^{1},{ }^{1}$ Xuhua Ren, ${ }^{1}$ and Yunrui Deng ${ }^{2}$ \\ ${ }^{1}$ College of Water Conservancy and Hydropower Engineering, Hohai University, Nanjing 210098, China \\ ${ }^{2}$ Power China Zhongnan Engineering Corporation Limited, Changsha 410000, China \\ Correspondence should be addressed to Jixun Zhang; zhangjixun@hhu.edu.cn
}

Received 26 May 2021; Revised 23 June 2021; Accepted 31 July 2021; Published 9 August 2021

Academic Editor: Qilong Xue

Copyright (c) 2021 Jiawei Lu et al. This is an open access article distributed under the Creative Commons Attribution License, which permits unrestricted use, distribution, and reproduction in any medium, provided the original work is properly cited.

The cohesion $c$ and internal friction angle $\varphi$ play different roles in the progressive failure process of the slope, which indicates that the reduction factors $k_{c}$ and $k_{\varphi}$ should be different in the calculation. Based on this, the program of double strength reduction factor method was compiled with FISH language, in order to study its application in the rock slopes under different distributions of weak interlayer, and the following conclusions were drawn: (1) the plastic zone calculated by double strength reduction factor method is generally distributed in the weak interlayer, which is basically consistent with the calculation result of the traditional method; (2) the degree to which $c$ and $\varphi$ play a role is related to the inclination angle of the bottom sliding surface of the unstable block $\theta$. If $\theta<45^{\circ}, \varphi$ will play a greater role. If $\theta \geq 45^{\circ}, c$ will play a greater role; (3) according to the "Pan's principle," the matching reduction principle of " $k_{c}>k_{\varphi}$ " can be adopted when $\theta<45^{\circ}$, and the matching reduction principle of " $k_{c}<k_{\varphi}$ " can be adopted when $\theta \geq 45^{\circ}$; (4) the definition of the comprehensive safety factor " $K_{2}$ " in the text is more suitable for the application of double strength reduction factor method in the stability analysis of rock slopes. The applicability of the above conclusions is verified by an actual engineering.

\section{Introduction}

At present, the commonly used methods for the stability analysis of slopes mainly include limit equilibrium method, strength reduction method, and overload method, etc., all of which have certain advantages and disadvantages [1-5]. The limit equilibrium method is simple and clear in calculation, but the sliding surface should be assumed in advance before calculation, while the strength reduction method and overload method make up for this defect, but the calculation efficiency of the strength reduction method is low, and the attenuation process of rock and soil strength parameters cannot be reflected in the overload method, which is not consistent with the actual engineering. For the rock slope, its stability is mainly controlled by the distribution of the internal structural plane and its mechanical properties [6-8]. In view of this characteristic, the strength reduction method is more and more widely used in the stability analysis of rock slopes.
The cohesion $c$ and internal friction angle $\varphi$ of the rock and soil mass are divided by the same reduction factor at the same time in the traditional strength reduction method. By continuously increasing the reduction factor, the slope reaches the critical failure state, and the reduction factor under the critical state is taken as the safety factor of the slope. However, considering the physical and mechanical mechanism, in the progressive failure process of the slope, the attenuation speed and degree of $c$ and $\varphi$ are different, and their contributions to the stability of the slope are also different [9]. If the same reduction factor is adopted, the respective safety reserves of $c$ and $\varphi$ cannot be truly reflected. Therefore, the double strength reduction factor method that is more conforming to the actual engineering is proposed. Compared with the traditional strength reduction method, two different reduction factors are used for $c$ and $\varphi$ in the double strength reduction factor method, which leads to two key issues: (1) What matching reduction principle should $c$ and $\varphi$ follow; (2) how to define the comprehensive safety 
factor of the slope. Related scholars have carried out research on double strength reduction factor method currently. In terms of matching reduction principle, Taylor [10] proposed firstly that when the sliding failure occurs on a slope, the frictional resistance is fully exerted firstly and then supplemented by the cohesion. In addition, the safety factor of the slope is defined as the ratio of the actual cohesion of the soil on the sliding surface to the cohesion needed to make the slope reach the critical failure state. This viewpoint implies that the reduction factor of internal friction angle is always 1. Later, Tang et al. [11, 12] carried out mechanism analysis for double strength reduction factor method and proposed the matching reduction principles for sand and clay. Yuan et al. [13] established the $c-\tan \varphi$ curve of the critical state of the slope under different slope shapes, assuming that the cohesion and internal friction angle are reduced along the shortest path of the critical curve. Zhu et al. [14] studied the application of double strength reduction factor method in cohesive soil slope with " $K=R 1 / R 2$ " as the matching reduction principle, where $R 1$ and $R 2$ were the reduction factor obtained by separately reducing the cohesion and internal friction angle. Jiang et al. [15] pointed out that the ratio between the reduction factor of cohesion and the reduction factor of internal friction angle should be 1.75 in an isotropic soil slope. Qi et al. [16] studied the relationship between the two reduction factors under the earthquake and calculated the dynamic stability safety factor of the slope. Yuan et al. [17] studied the proportional relationship between the two reduction factors under different slope shapes. Chen et al. $[18,19]$ introduced the equivalent influence angle which is defined as the slope angle at which $c$ and $\varphi$ share identical contributions to stability, and based on this, a new matching reduction principle and the definition of comprehensive safety factor are put forward. Xue et al. have deduced the matching reduction principles based on different soil softening assumptions [20, 21]. Regarding the definition of the comprehensive safety factor, related scholars have also put forward some different definitions. Tang et al. $[11,12,15]$ used the arithmetic average of the two reduction factors as the comprehensive safety factor of the slope, as shown in equation (2). Yuan et al. [17] proposed a new definition method based on the degree to which the cohesion and internal friction angle play a role in the stability of slopes at different slope angles, as shown in equation (3); Isakov and Moryachkov [22] proposed another typical definition based on the idea of the shortest strength reduction path, as shown in equation (4). Xue et al. [20] defined the comprehensive safety factor by taking the attenuation degree of the shear strength parameter of rock and soil mass or the contribution degree to the slope stability as the weight, but this definition method has great limitations and is not widely applicable.

Most of the above experts and scholars' studies on the double strength reduction factor method were based on the soil slope as the application object, and few involved the rock slope with weak interlayer. However, this type of rock slopes has poor stability and high hazard. Therefore, the double strength reduction factor method was applied to the stability analysis of this type of rock slopes, and the matching reduction principle and the definition of the comprehensive safety factor applicable to it were studied. It can not only reflect the respective roles and degrees of $c$ and $\varphi$ in the progressive failure process of the slope, but also fill the vacancy of double strength reduction factor method in the engineering application, which is very necessary. Based on this, this paper used the built-in FISH language in the software FLAC ${ }^{3 \mathrm{D}}$ to compile the program of double strength reduction factor method and calculated the reduction factor of cohesion, reduction factor of internal friction angle, and three typical comprehensive safety factors of the rock slope under different distributions of weak interlayer. The matching reduction principle and the definition of comprehensive safety factor for such type of rock slopes were studied and established.

\section{Double Strength Reduction Factor Method}

2.1. Fundamental Theory. In the traditional strength reduction method, the shear strength parameters $c$ and $\varphi$ of rock and soil mass are reduced synchronously by the same reduction factor. However, in the progressive failure process of the slope, the attenuation speed and degree of $c$ and $\varphi$ are different. In order to conform to the actual engineering, different reduction factors should be used for reduction, that is, the double strength reduction factor method. The shear strength parameters after reduction are expressed as follows:

$$
\left\{\begin{array}{l}
\tan \varphi_{m}=\frac{\tan \varphi}{k_{\phi}} \\
c_{m}=\frac{c}{k_{c}}
\end{array}\right.
$$

where $c$ and $\varphi$ are the shear strength provided by the rock and soil mass itself; $c_{m}$ and $\varphi_{m}$ are the shear strength of rock and soil mass required in the critical failure state; $k_{\varphi}$ is the reduction factor of internal friction angle; $k_{c}$ is the reduction factor of cohesion.

Due to groundwater, physical and chemical erosion, and other reasons, the strength parameter of rock and soil mass will attenuate accordingly. When the attenuation reaches a certain level, the rock slope with weak interlayer will generally slide along the interlayer zone. In the progressive failure process of the slope, if the internal friction angle $\varphi$ attenuates faster and reduces much than the $\operatorname{cohesion} c$, then the reduction factor of internal friction angle $k_{\varphi}$ is greater than the reduction factor of cohesion $k_{c}$, that is, the more fully the cohesion plays its role. On the contrary, if the cohesion $c$ attenuates faster and reduces much more than the internal friction angle $\varphi$, then the reduction factor of cohesion $k_{c}$ is greater than the reduction factor of internal friction angle $k_{\varphi}$, that is, the more fully the internal friction angle plays its role.

2.2. Definition of Comprehensive Safety Factor. The safety factor is an important index to evaluate whether the slope is safe and stable, and the double reduction factor method uses 
two different reduction factors to reveal the progressive failure process of the slope, so how to reasonably convert the two reduction factors into the comprehensive safety factor of the slope is crucial. The current mainstream definitions of comprehensive safety factor at home and abroad include the following three types:

$$
\begin{aligned}
& K_{1}=\frac{k_{c}+k_{\varphi}}{2}, \\
& K_{2}=\frac{\sqrt{2} k_{c} k_{\varphi}}{\sqrt{k_{c}^{2}+k_{\varphi}^{2}}} \\
& \left\{\begin{array}{l}
R=\sqrt{\left(1-\frac{1}{k_{c}}\right)^{2}+\left(1-\frac{1}{k_{\varphi}}\right)^{2}}, \\
K_{3}=\frac{1}{(1-R) / \sqrt{2}} .
\end{array}\right.
\end{aligned}
$$

The relationship between the two reduction factors and the comprehensive safety factor is complex, so there is no unified understanding of which way to define it. Therefore, this paper calculated the above three comprehensive safety factors and then conducted a comparative analysis to study which definition is more suitable for the application of double strength reduction factor method in the stability analysis of rock slopes.

2.3. The Thinking of Programming. Since the double strength reduction factor method uses two different reduction factors, there are numerous combinations of $c_{m}$ and $\varphi_{m}$ that make the slope reach the critical failure state. In order to avoid the blindness of the values of $k_{\varphi}$ and $k_{c}$, a proportionality coefficient $k$ was defined:

$$
k=\frac{k_{c}}{k_{\varphi}} .
$$

The author used the built-in FISH language in the software FLAC ${ }^{3 \mathrm{D}}$ to compile the program of double strength reduction factor method. The main thinking of the program is shown in Figure 1.

$l$ is the value assigned to the proportionality coefficient; $m$ and $n$ are, respectively, assigned the increment and initial value of the reduction factor of internal friction angle; mech.ratio is the force unbalance ratio; the remaining variables have the same meaning as the variables in the above equations.

In the process of calculating and solving, the reduction factor of cohesion and internal friction angle and the comprehensive safety factor of the slope can be obtained under different matching reduction principles only by assigning different values of the proportionality coefficient $k$. In order to improve the calculation efficiency, the value of $\Delta k$ can be set as 1 to obtain the approximate range of $k_{\varphi}$ firstly, and then the precise value of $k_{\varphi}$ can be obtained by gradually reducing $\Delta k$.

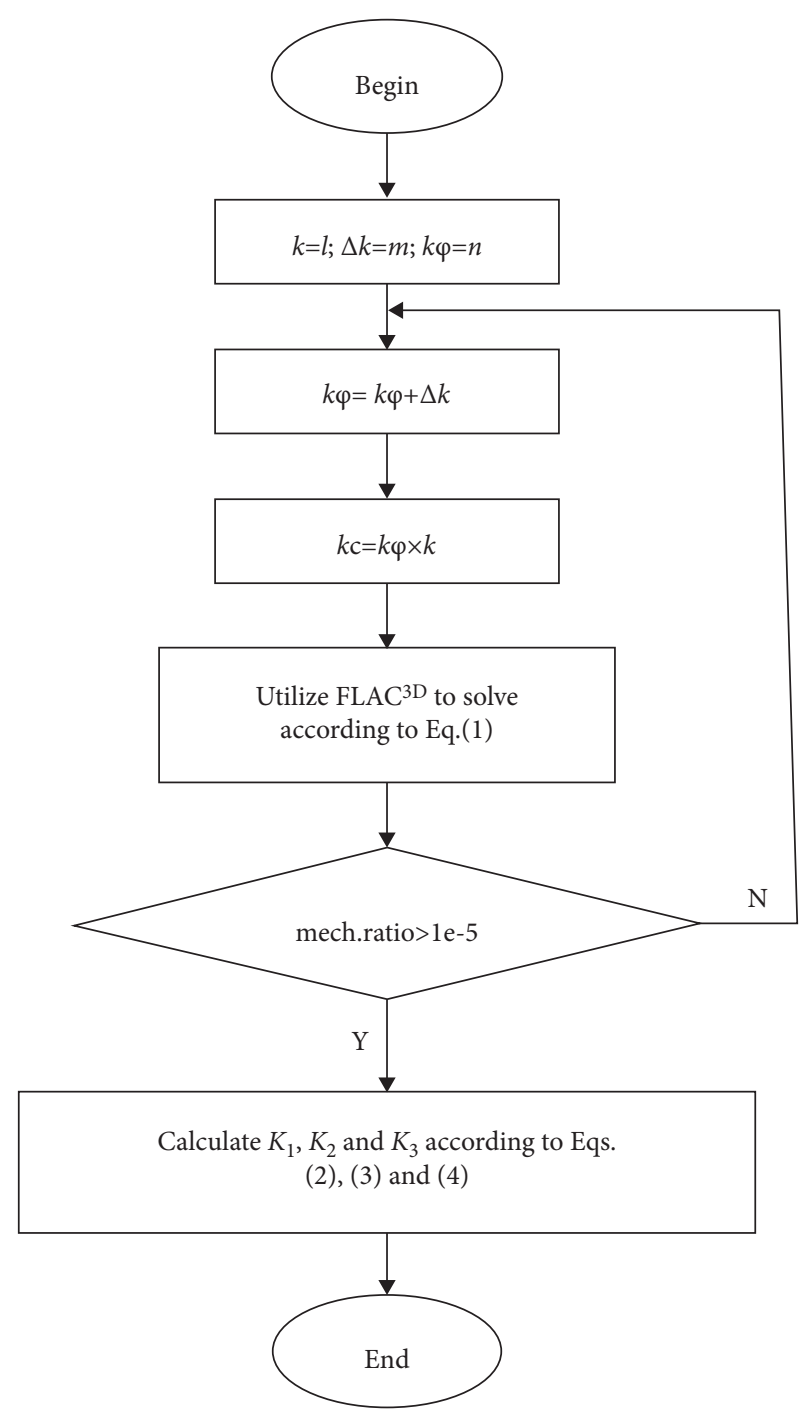

Figure 1: The solution flow chart of double strength reduction factor method.

\section{Numerical Experiment}

3.1. Calculation Model and Material Parameters. According to the influence of the boundary range of the model proposed in [23] on the calculation accuracy, a rock slope with height $H=50 \mathrm{~m}$ and slope angle $\alpha=72^{\circ}$ was established. In order to study the application of double strength reduction factor method in the case of different distributions of weak interlayer, single weak interlayer and combined weak interlayer with different inclination angle $\theta$ $(\beta, \gamma)$ were set up in the slope. The schematic diagram of the model is shown in Figures 2(a) and 2(b), and the material parameters of rock mass and weak interlayer are shown in Table 1.

3.2. Experimental Scheme. In this experiment, the program of double strength reduction factor method was used to calculate the two types of rock slopes with single weak interlayer and combined weak interlayer. For the experimental 


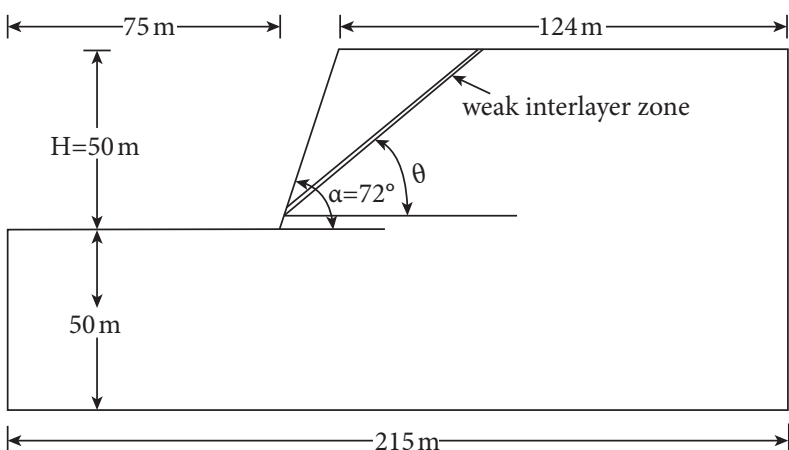

(a)

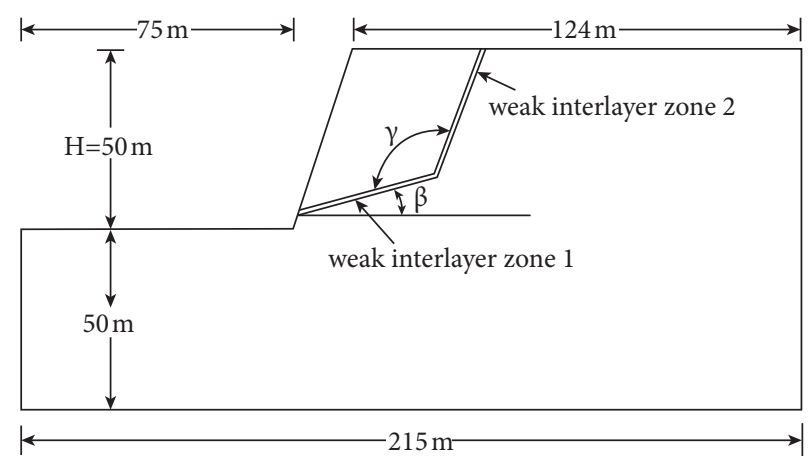

(b)

Figure 2: Schematic diagram of model. (a) Single weak interlayer zone. (b) Combined weak interlayer zone.

TABLE 1: Mechanical parameters of materials.

\begin{tabular}{lccccc}
\hline Material & $E(\mathrm{GPa})$ & $v$ & $\rho\left(\mathrm{g} \cdot \mathrm{cm}^{-3}\right)$ & $c(\mathrm{kPa})$ & $\varphi\left(^{\circ}\right)$ \\
\hline Rock mass & 18 & 0.22 & 2.75 & 1300 & 45 \\
Weak interlayer & 1 & 0.38 & 2 & 150 & 25 \\
\hline
\end{tabular}

group with single weak interlayer, the five schemes $\theta=30^{\circ}$, $35^{\circ}, 40^{\circ}, 45^{\circ}$, and $50^{\circ}$ were selected, which were referred to as T1 experimental group (a-e). For the experimental group with combined weak interlayer, the five schemes of $\beta=15^{\circ}$, $\gamma=90^{\circ}, 105^{\circ}, 120^{\circ}, 130^{\circ}$, and $140^{\circ}$ were selected, and another scheme of $\beta=45^{\circ}, \gamma=135^{\circ}$ was added for comparative analysis, which were referred to as T2 experimental group (a-f). According to the above schemes, the reduction factor of cohesion and internal friction angle and the three definitions of the comprehensive safety factor were, respectively, calculated when the proportionality coefficient $k=0.5,0.6$, $0.7,0.8,0.9,1.0,1.2,1.4,1.6,1.8$, and 2.0 . The specific experimental schemes were shown in Table 2. According to the fundamental theory of double strength reduction factor method, when the reduction coefficient $k=1$, it is equivalent to the traditional strength reduction method.

\subsection{Analysis of Experimental Results}

3.3.1. T1 Experimental Group. The plastic zones of the rock slopes of each scheme in the T1 experimental group are basically distributed in the weak interlayer zone; that is, no matter how the inclination angle $\theta$ of the weak interlayer and the proportional coefficient $k$ change, most of this type of rock slopes will slide along the weak interlayer zone. Due to space limitations, this paper only enumerates the distribution of the plastic zone with scheme $\theta=40^{\circ}$, as shown in Figure 3.

The $\left(k_{c}, k_{\varphi}, K_{1}, K_{2}, K_{3}\right)-k$ curve of each scheme in the T1 experimental group is shown in Figures $4(\mathrm{a})-4(\mathrm{e})$. It can be seen from the figure that when $k=1, k_{c}=k_{\varphi}=K_{1}=K_{2}=K_{3}$ for each scheme of the experimental group, which indicates that the comprehensive safety factor calculated according to equations (2)-(4) is consistent when calculated by the traditional strength reduction method. When $k \neq 1$, $K_{2}<K_{1}<K_{3}$, indicating that the result of the comprehensive
TABLe 2: Experimental schemes.

\begin{tabular}{lccccc}
\hline \multicolumn{2}{c}{ T1 } & \multicolumn{5}{c}{$\mathrm{T} 2$} \\
Scheme & $\theta\left(^{\circ}\right)$ & Scheme & $\beta\left({ }^{\circ}\right)$ & $\gamma\left({ }^{\circ}\right)$ & $k$ \\
\hline (a) & 30 & (a) & 15 & 90 & $0.5,0.6$ \\
(b) & 35 & (b) & 15 & 105 & $0.7,0.8$ \\
(c) & 40 & (c) & 15 & 120 & $0.9,1.0$ \\
(d) & 45 & (d) & 15 & 130 & $1.2,1.4$ \\
(e) & 50 & (e) & 15 & 140 & $1.6,1.8$ \\
& & (f) & 45 & 135 & 2.0 \\
\hline
\end{tabular}

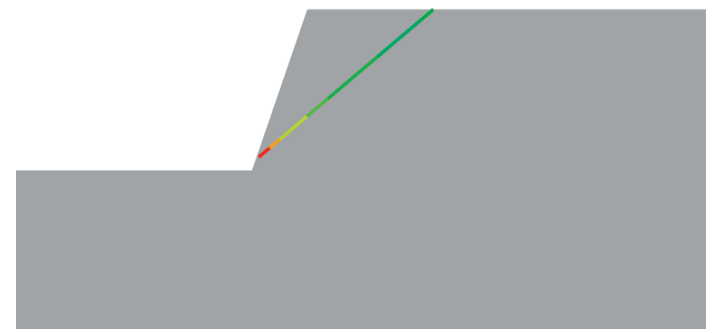

FIGURE 3: Distribution of plastic zone in the T1 experimental group.

safety factor calculated according to equation (3) is the most conservative. In addition, $K_{1}, K_{2}$, and $K_{3}$ have different variation trends with the increase of the proportionality coefficient $k$. Noting that the corresponding values of $k$ when $K_{1}, K_{2}$, and $K_{3}$ take the minimum (maximum) value are $k_{1 \text { min }}, k_{2 \max }$, and $k_{3 \min }$. The values of $k_{1 \text { min }}, k_{2 \max }$, and $k_{3 \min }$ for each scheme in the T1 experimental group were shown in Table 3. $K_{1}$ decreases first and then increases with the increase of $k, k_{1 \min }$ increases with the increase of the inclination angle $\theta$, and when $\theta<45^{\circ}, k_{1 \min }<1$, and when $\theta \geq 45^{\circ}$, $k_{1 \text { min }}>1 . K_{2}$ increases first and then decreases with the increase of $k, k_{2 \max }$ decreases with the increase of $\theta$, and when $\theta<45^{\circ}, k_{2 \max }>1$, and when $\theta \geq 45^{\circ}, k_{2 \max }<1$. The variation trend of $K_{3}$ is basically similar to that of $K_{1}$, but its variation range is larger.

Although the variation trend of $K_{1}, K_{2}$, and $K_{3}$ with $k$ is not consistent, they all reveal the same law. Note that the comprehensive safety factors calculated when $k=1$ are $K_{1 c}$, $K_{2 c}$, and $K_{3 c}$. If $\theta<45^{\circ}$, when $k<1$, there are $K_{1}<K_{1 c}$, $K_{2}<K_{2 c}$, and $K_{3}<K_{3 c}$, and when $k>1, K_{1}>K_{1 c}, K_{2}>K_{2 c}$, 

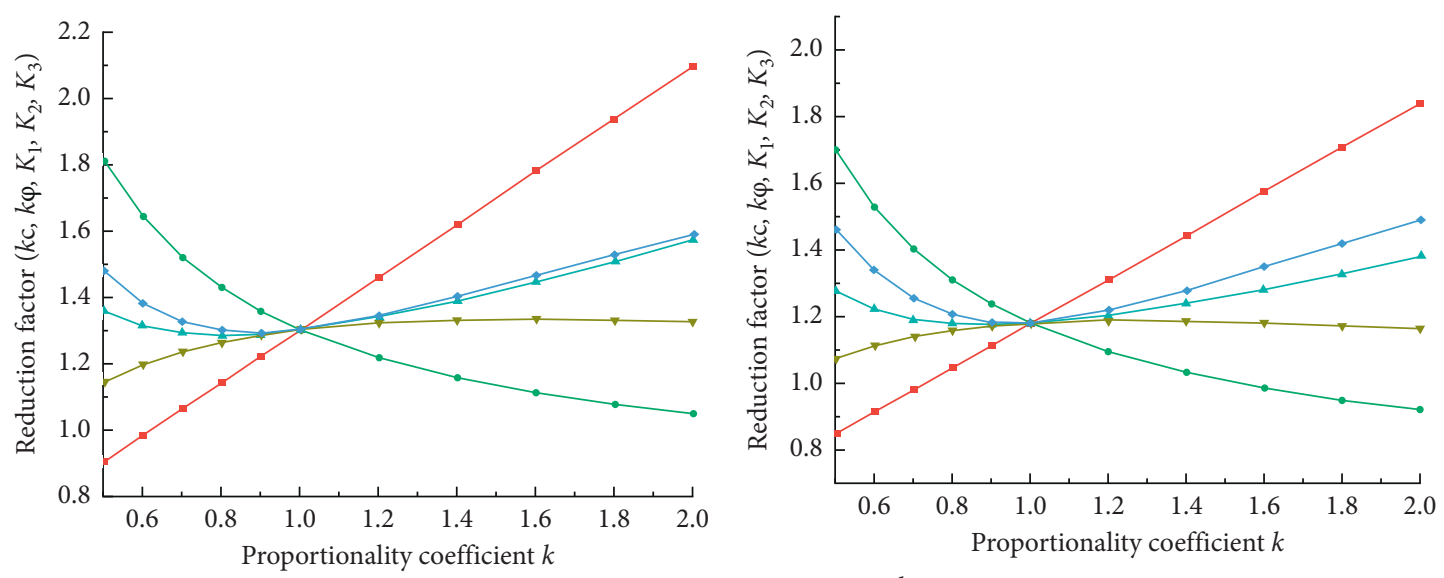

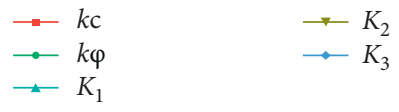

(a)

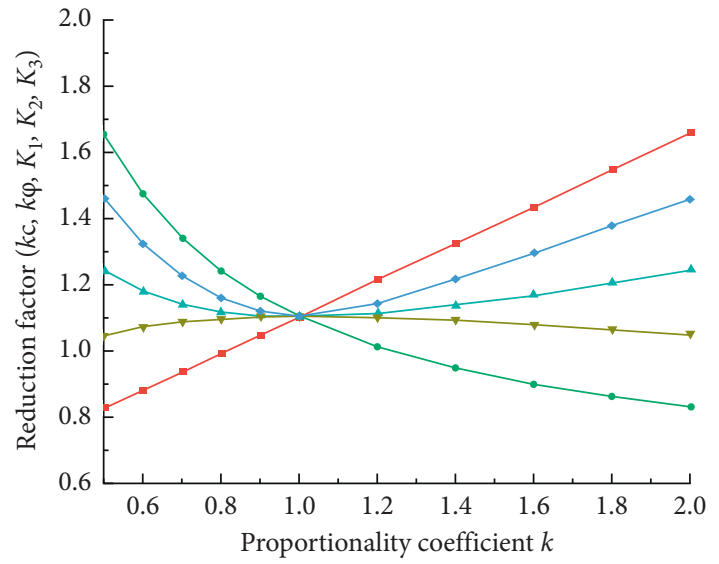

$$
\begin{array}{ll}
\rightarrow k \mathrm{c} & \rightarrow K_{2} \\
\rightarrow k \varphi & \rightarrow K_{3}
\end{array}
$$

(c)

$$
\begin{array}{ll}
\because k \mathrm{c} & \because K_{2} \\
\because k \varphi & \because K_{3}
\end{array}
$$

(b)

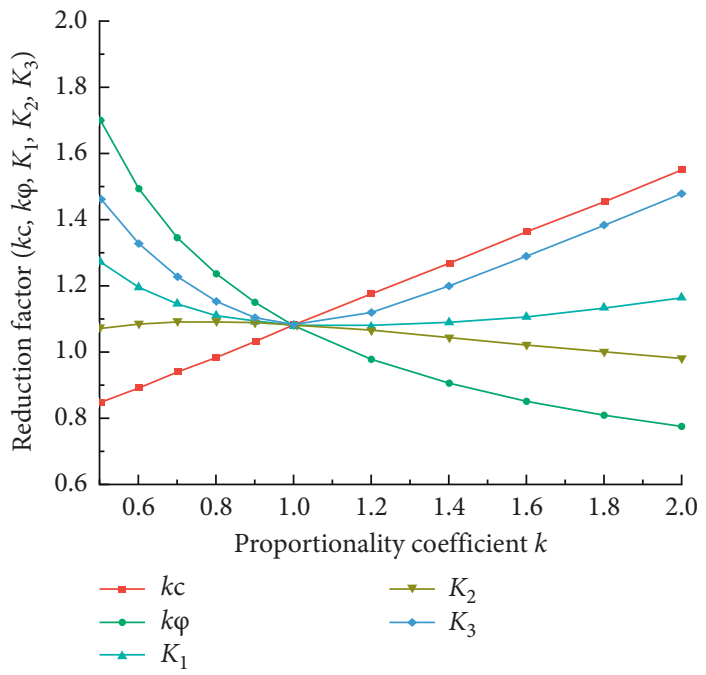

(d)

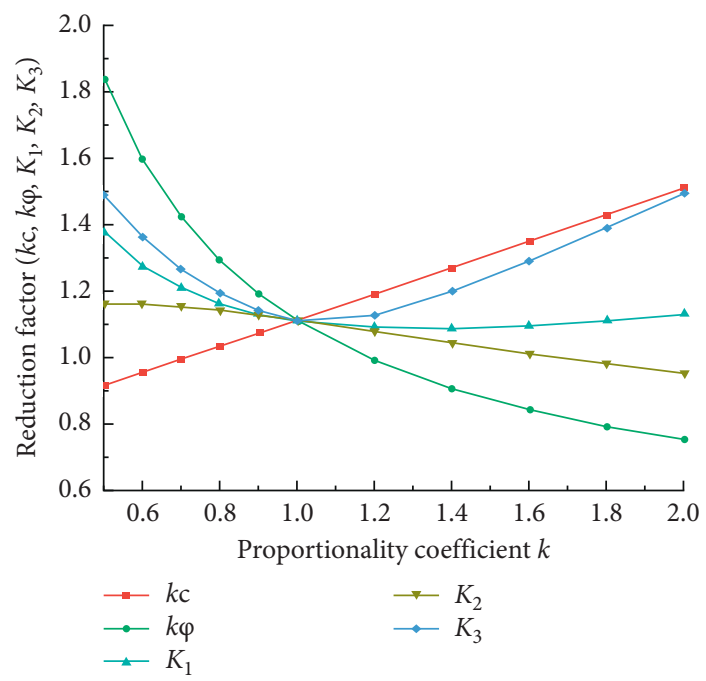

(e)

Figure 4: The $\left(k_{c}, k_{\varphi}, K_{1}, K_{2}, K_{3}\right)-k$ curve of T1 experimental group. (a) $\theta=30^{\circ}$. (b) $\theta=35^{\circ}$. (c) $\theta=40^{\circ}$. (d) $\theta=45^{\circ}$. (e) $\theta=50^{\circ}$. 
TABLE 3: The values of $k_{1 \min }, k_{2 \max }$, and $k_{3 \min }$ for each scheme in the T1 experimental group.

\begin{tabular}{lccc}
\hline$\theta\left(^{\circ}\right)$ & $k_{1 \min }$ & $k_{2 \max }$ & $k_{3 \min }$ \\
\hline 30 & 0.8 & 1.6 & 0.8 \\
35 & 0.9 & 1.4 & 0.9 \\
40 & 0.9 & 1.2 & 1.0 \\
45 & 1.2 & 0.7 & 1.0 \\
50 & 1.4 & 0.5 & 1.0 \\
\hline
\end{tabular}

and $K_{3}>K_{3 c}$. If $\theta \geq 45^{\circ}$, when $k<1, K_{1}>K_{1 c}, K_{2}>K_{2 c}$, and $K_{3}>K_{3 c}$, and when $k>1$, there are $K_{1}<K_{1 c}, K_{2}<K_{2 c}$, and $K_{3}<K_{3 c}$. In other words, when the inclination angle of the weak interlayer $\theta<45^{\circ}$, the comprehensive safety factor of the slope calculated by the matching reduction principle of " $k_{c}>k_{\varphi}$ " is generally larger than that calculated by the traditional strength reduction method. The comprehensive safety factor of the slope calculated by the matching reduction principle of " $k_{c}<k_{\varphi}$ " is generally smaller than that calculated by the traditional strength reduction method. However, when the inclination angle $\theta \geq 45^{\circ}$, the law is opposite. The main reason for the above phenomenon is that the weak interlayer zone is the bottom sliding surface of the unstable block sliding downwards; if the inclination angle $\theta$ is smaller, the normal stress acting on the bottom sliding surface will be larger, the friction resistance $\sigma \tan \varphi$ acting on the sliding surface will be larger, and the internal friction angle will play a greater role. Therefore, the matching principle with a smaller $k_{\varphi}$ can produce larger sliding resistance, and the calculated safety factor will be larger. On the contrary, if $\theta$ is larger, the normal stress acting on the bottom sliding surface will be smaller, the friction resistance $\sigma \tan \varphi$ will be smaller, and the internal friction angle will play a smaller role. In this case, the matching principle with a smaller $k_{\varphi}$ can not produce larger sliding resistance, and the calculated safety factor value will be smaller.

3.3.2. T2 Experimental Group. The plastic zones of the rock slopes of each scheme in the T2 experimental group are also basically distributed in the weak interlayer zone; that is, the failure mode of this type of rock slope is mainly the sliding failure of the unstable block surrounded by various weak interlayer zones along the plastic through plane. Due to space limitations, this paper only enumerates the distribution of the plastic zone with scheme $\beta=15^{\circ}, \gamma=130^{\circ}$, as shown in Figure 5.

The $\left(k_{c}, k_{\varphi}, K_{1}, K_{2}, K_{3}\right)-k$ curve of each scheme in the T2 experimental group is shown in Figures 6(a)-6(f). The values of $k_{1 \min }, k_{2 \max }$, and $k_{3 \min }$ for each scheme in the $\mathrm{T} 2$ experimental group were shown in Table 4 . It can be seen from the figure that when $k=1, k_{c}=k_{\varphi}=K_{1}=K_{2}=K_{3}$ for each scheme of the experimental group, which indicates that the comprehensive safety factor calculated according to equations (2)-(4) is still consistent when calculated by the traditional strength reduction method. However, when $k \neq 1$, for the scheme (a-e), $K_{2}<K_{3}<K_{1}$, and for the scheme (f), $K_{2}<K_{3}<K_{1}$. Similarly, the calculation result of $K_{2}$ is also the most conservative. In addition, the variation trend

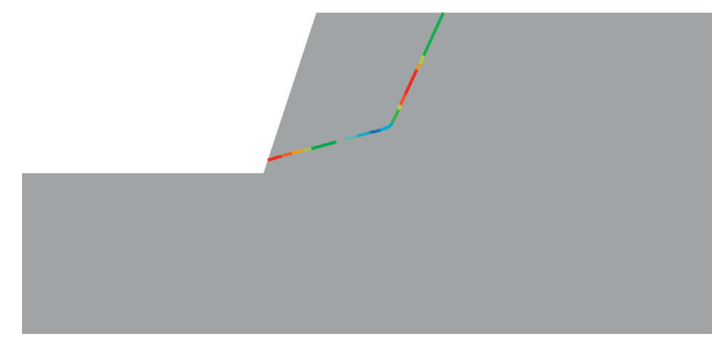

FIgURE 5: Distribution of plastic zone in the T2 experimental group.

of $K_{2}$ in scheme (a-e) is basically similar to that of scheme $(\mathrm{a}-\mathrm{c})$ in the $\mathrm{T} 1$ experimental group, but the variation trend of $K_{1}$ and $K_{3}$ is slightly changed, which increases with the increase of the proportionality coefficient $k$. The variation trend of $K_{1}, K_{2}$, and $K_{3}$ in scheme (f) is roughly similar to that of scheme $(\mathrm{d}-\mathrm{f})$ in the $\mathrm{T} 1$ experimental group, and the calculated values of $K_{1}, K_{2}$, and $K_{3}$ in this scheme are also far less than those of the other five schemes. The above calculation results show that the stability of this type of rock slope and the variation trend of $K_{1}, K_{2}$, and $K_{3}$ with the proportionality coefficient $k$ are mainly controlled by the inclination angle $\beta$ of the weak interlayer zone 1 and have little relationship with the angle $\gamma$ between the two weak interlayer zones. In addition, combined with the calculation results of each scheme in the $\mathrm{T} 1$ and $\mathrm{T} 2$ experimental groups, it can be seen that the numerical value of $k_{c}$ and $k_{\varphi}$ has a greater impact on $K_{1}$ and $K_{3}$, while the impact on $K_{2}$ is less. If $k_{c}$ or $k_{\varphi}$ is too small, the calculation results of $K_{1}$ and $K_{3}$ will be too large, and when the proportionality coefficient $k$ is small or large, the calculation results will be distorted.

The weak interlayer zones 1 and 2 marked in Figure 2(b) are generally referred to as the bottom sliding surface and trailing edge pull-fracturing surface of the unstable block in engineering applications. By comparing Figures 6(a)-6(f), it can be seen that if the inclination angle of the bottom sliding surface $\beta<45^{\circ}$, when $k<1$, there are $K_{1}<K_{1 c}$, $K_{2}<K_{2 c}$, and $K_{3}<K_{3 c}$; when $k>1, K_{1}>K_{1 c}, K_{2}>K_{2 c}$, and $K_{3}>K_{3 c} ;$ if $\beta \geq 45^{\circ}$, when $k<1, K_{1}>K_{1 C}, K_{2}>K_{2 C}$, and $K_{3}>K_{3 C}$; when $k>1$, there are $K_{1}<K_{1 C}, K_{2}<K_{2 C}$, and $K_{3}<K_{3 C}$. It is the same as the law revealed by the T1 experimental group, but in the process of sliding failure of the rock slopes in the T2 experimental group, the degree to which the internal friction angle and cohesion play a role mainly depends on the inclination angle $\beta$ of the bottom sliding surface, while the angle $\gamma$ between the bottom sliding surface and the trailing edge surface has little effect. The reason is that the inclination angle and shear strength of the bottom sliding surface are the main factors that determine whether the sliding failure of the unstable block occurs, and the trailing edge pull-fracturing surface is only formed when the unstable block slides downward and cuts along the path with the least sliding resistance. As for the different effects of internal friction angle and cohesion in the failure process of each rock slope in the T2 experimental group, the reasons are the same as above, which will not be repeated here. 

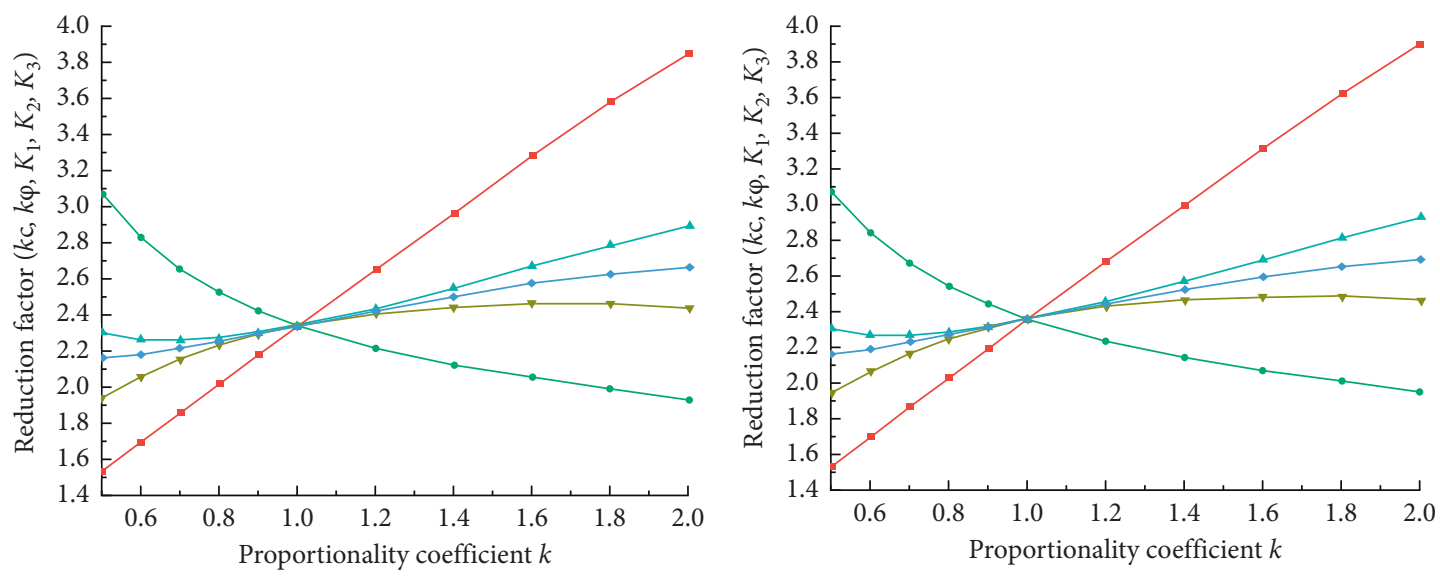

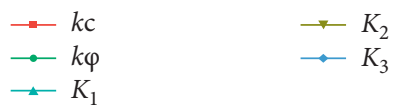

(a)

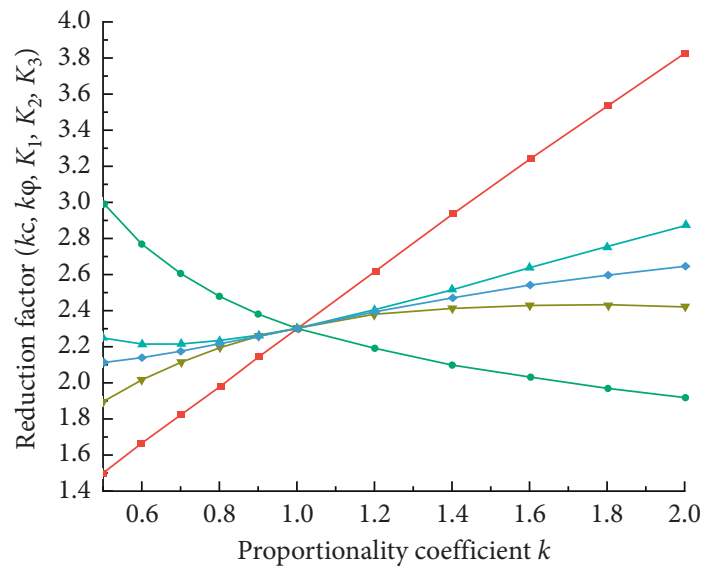

(c)

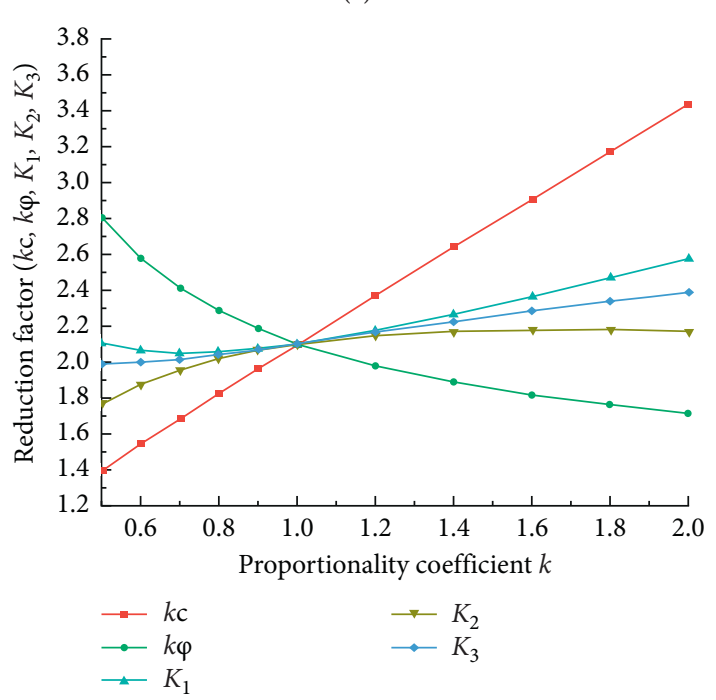

(e)

$$
\begin{aligned}
& \rightarrow K_{2} \\
& \rightarrow-K_{3}
\end{aligned}
$$

$$
\begin{array}{ll}
\because k \mathrm{c} & \rightarrow K_{2} \\
\rightarrow k \varphi & \rightarrow-K_{3} \\
-K_{1} &
\end{array}
$$

(b)

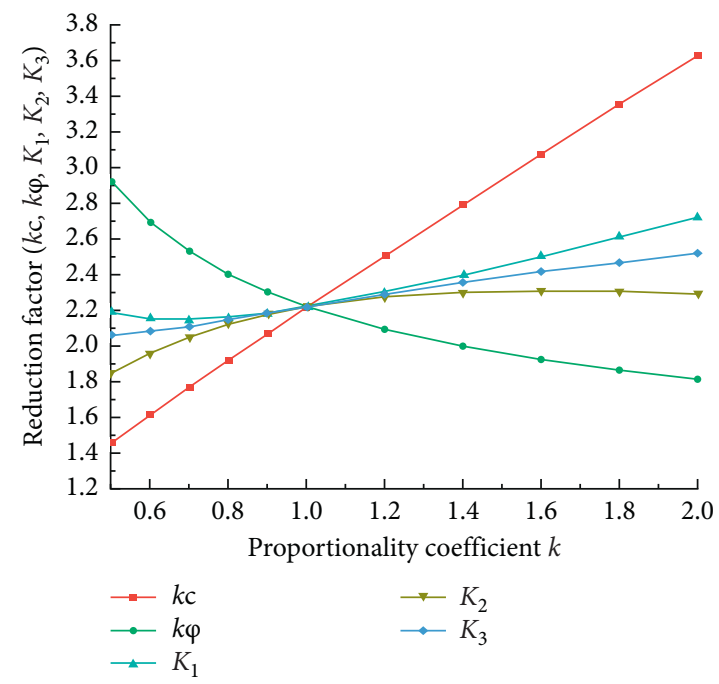

(d)

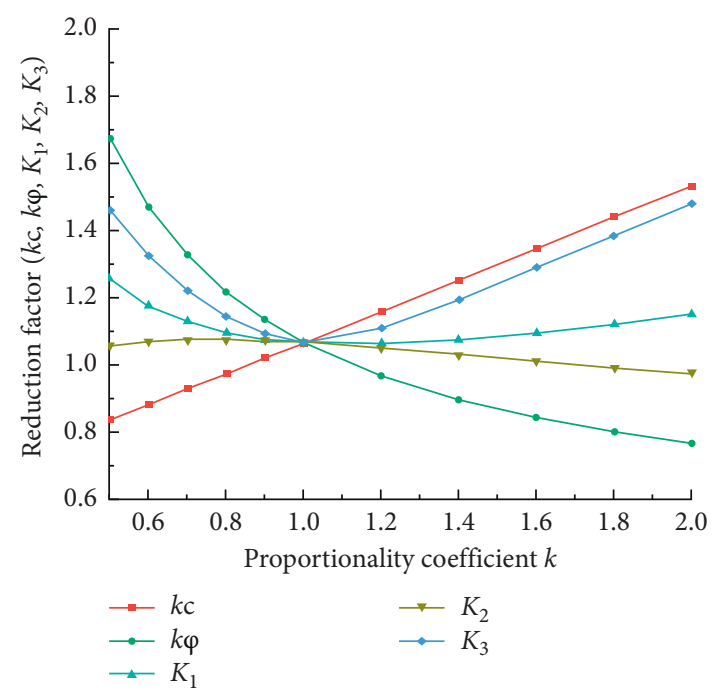

(f)

Figure 6: The $\left(k_{c}, k_{\varphi}, K_{1}, K_{2}, K_{3}\right)-k$ curve of T2 experimental group. (a) $\beta=15^{\circ}, \gamma=90^{\circ}$. (b) $\beta=15^{\circ}, \gamma=105^{\circ}$. (c) $\beta=15^{\circ}, \gamma=120^{\circ}$. (d) $\beta=15^{\circ}$, $\gamma=130^{\circ}$. (e) $\beta=15^{\circ}, \gamma=140^{\circ}$. (f) $\beta=45^{\circ}, \gamma=135^{\circ}$. 
TABLE 4: The values of $k_{1 \min }, k_{2 \max }$, and $k_{3 \min }$ for each scheme in the T2 experimental group.

\begin{tabular}{lcccc}
\hline$\beta\left({ }^{\circ}\right)$ & $\gamma\left({ }^{\circ}\right)$ & $k_{1 \min }$ & $k_{2 \max }$ & $k_{3 \min }$ \\
\hline 15 & 90 & 0.7 & 1.6 & 0.5 \\
15 & 105 & 0.7 & 1.6 & 0.5 \\
15 & 120 & 0.7 & 1.6 & 0.5 \\
15 & 130 & 0.7 & 1.6 & 0.5 \\
15 & 140 & 0.7 & 1.6 & 0.5 \\
45 & 135 & 1.2 & 0.8 & 1.0 \\
\hline
\end{tabular}

3.4. Discussion. Combined with the calculation results of $\mathrm{T} 1$ and T2 numerical experiments, it can be seen that the calculation results of $K_{2}$ are the most conservative among the three definitions of the comprehensive safety factor, and the variation trend of $K_{2}$ with the proportional coefficient $k$ is less affected by the numerical size of $k_{c}$ and $k_{\varphi}$. Secondly, when the proportionality coefficient $k$ is too small or large, the calculation results of $K_{2}$ can still follow the general law without numerical mutation. Therefore, the definition of $K_{2}$ is more suitable for the application of double strength reduction factor method in the stability analysis of rock slope.

In addition, in the progressive failure process of the rock slope, the degree to which the internal friction angle and cohesion play a role is mainly related to the inclination angle of the bottom sliding surface of the unstable block. If the inclination angle is less than $45^{\circ}$, the internal friction angle plays a greater role, and if the inclination angle is greater than or equal to $45^{\circ}$, the cohesion plays a greater role. Secondly, according to "Pan's principle," when the unstable block slides along a certain sliding surface, its internal force will automatically adjust to maximize the sliding resistance [24]. Based on this, when the inclination angle of the bottom sliding surface is less than $45^{\circ}$, the matching reduction principle of " $k_{c}>k_{\varphi}$ " can be adopted; when the inclination angle is more than $45^{\circ}$, the matching reduction principle of " $k_{c}<k_{\varphi}$ " can be adopted.

\section{Engineering Application}

4.1. Engineering Situation. The mountains on both banks of a dam site in the upper reaches of the Jinsha River are steep, with steep cliffs below the elevation of about $2100 \mathrm{~m}$, and the average topographic slope is about $68^{\circ}$, while the average topographic slope above the elevation is about $52^{\circ}$. There is no regional active fault distribution in the engineering area. Structural planes of level II and above mainly have faults $f_{203}$, and structural planes of level III have $f_{202}, f_{204}, f_{205}, f_{206}, f_{217}$, and $f_{218}$, and the small faults are relatively developed. The geological conditions of the dam site area are complex, and there are slate and carbonaceous limestone sandwiched in the limestone strata on the right bank. The geomorphology is shown in Figure 7.

4.2. Mesh Model and Material Parameters. A typical section $0+040$ of the right bank slope was selected for calculation and analysis. This section mainly contains weak structural planes $f_{202}, f_{203}, F_{218}, f_{1}$, and $f_{2}$, and its distribution is shown in Figure 8. The establishment of the mesh model is shown in

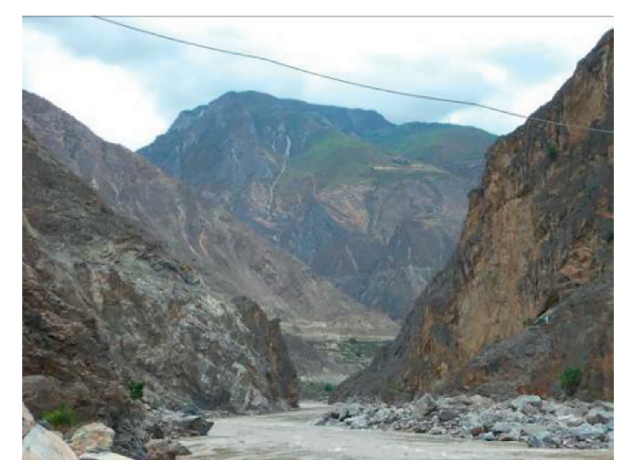

FIGURE 7: Geomorphology of the dam site area.

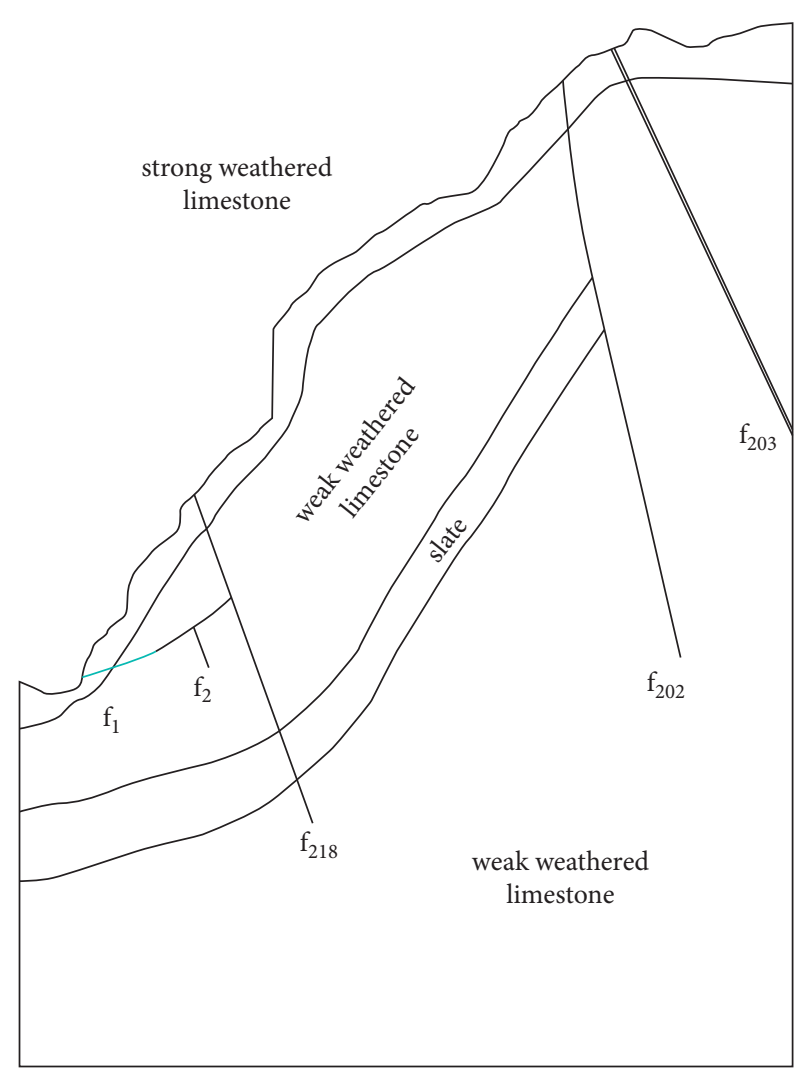

Figure 8: The section of $0+040$.

Figure 9, which was divided into 3942 elements, and the weak structural plane in the model was simulated by solid thin layer elements. The Cartesian coordinate system was selected for this calculation. The vertical direction is the $Z$ direction, the horizontal direction is the $X$ direction, and the vertical plane direction is the $Y$ direction. The boundary conditions for calculation were set as follows: constraining the displacement of the bottom boundary of the model in $X$, $Y$, and $Z$ directions, the displacement of the left and right sides in the $X$ direction, and the displacement of the front and back sides in the $Y$ direction. The material parameters of this calculation are shown in Table 5 .

4.3. Analysis of Calculation Results. When the calculation was not convergent, the development of the plastic zone of 


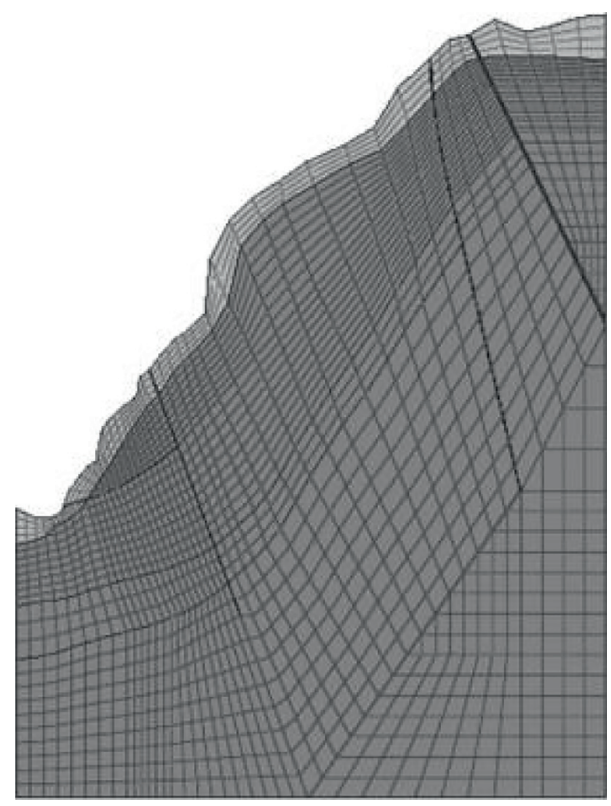

FIGURE 9: The mesh of the slope.

TABLE 5: Mechanical parameters of materials.

\begin{tabular}{lccccc}
\hline Material & $\begin{array}{c}E \\
(\mathrm{GPa})\end{array}$ & $v$ & $\begin{array}{c}\rho \\
\left(\mathrm{g} \cdot \mathrm{cm}^{-3}\right)\end{array}$ & $\begin{array}{c}c \\
(\mathrm{kPa})\end{array}$ & $\varphi\left(^{\circ}\right)$ \\
\hline $\begin{array}{l}\text { Strong weathered } \\
\text { limestone }\end{array}$ & 20 & 0.24 & 2.75 & 1300 & 45.0 \\
$\begin{array}{l}\text { Weak weathered } \\
\text { limestone }\end{array}$ & 22 & 0.22 & 2.75 & 1400 & 47.7 \\
Slate & 6 & 0.30 & 2.73 & 800 & 40.4 \\
$f_{202}$ & 0.5 & 0.40 & 1.90 & 200 & 26.6 \\
$f_{203}$ & 0.5 & 0.40 & 1.90 & 200 & 26.6 \\
$f_{218}$ & 1 & 0.40 & 1.90 & 200 & 27.5 \\
$f_{1}$ & 1 & 0.39 & 2.00 & 750 & 42.0 \\
$f_{2}$ & 1 & 0.39 & 2.00 & 150 & 26.6 \\
\hline
\end{tabular}

the rock slope model is shown in Figure 10. According to the figure, the weak interlayers $f_{1}$ and $f_{2}$ are connected with the plastic zone at $f_{218}$, forming a thorough sliding surface, in which $f_{1}$ and $f_{2}$ are the bottom sliding surfaces of the unstable block, and $f_{218}$ is the trailing edge pull-fracturing surface. Since the inclination angles of the bottom sliding surfaces $f_{1}$ and $f_{2}$ are less than $45^{\circ}$, according to the above analysis, the internal friction angle plays a greater role in the progressive failure process of the slope in this case. Therefore, the matching reduction principle of " $k_{c}>k_{\varphi}$ " is adopted based on "Pan's principle." The safety factors of the rock slope under different proportionality coefficient are summarized in Table 6. According to the calculation results, when the proportionality coefficient $k<1$, the error is negative, while when $k>1$, the error is positive, which confirms the conclusion drawn above. In addition, it can be seen that the absolute value of the error corresponding to $k<1$ is much larger than that when $k>1$, which further indicates that the internal friction angle plays a more obvious role in the progressive failure process of the rock slope. Therefore, the impact on the safety factor is also greater. On the basis of

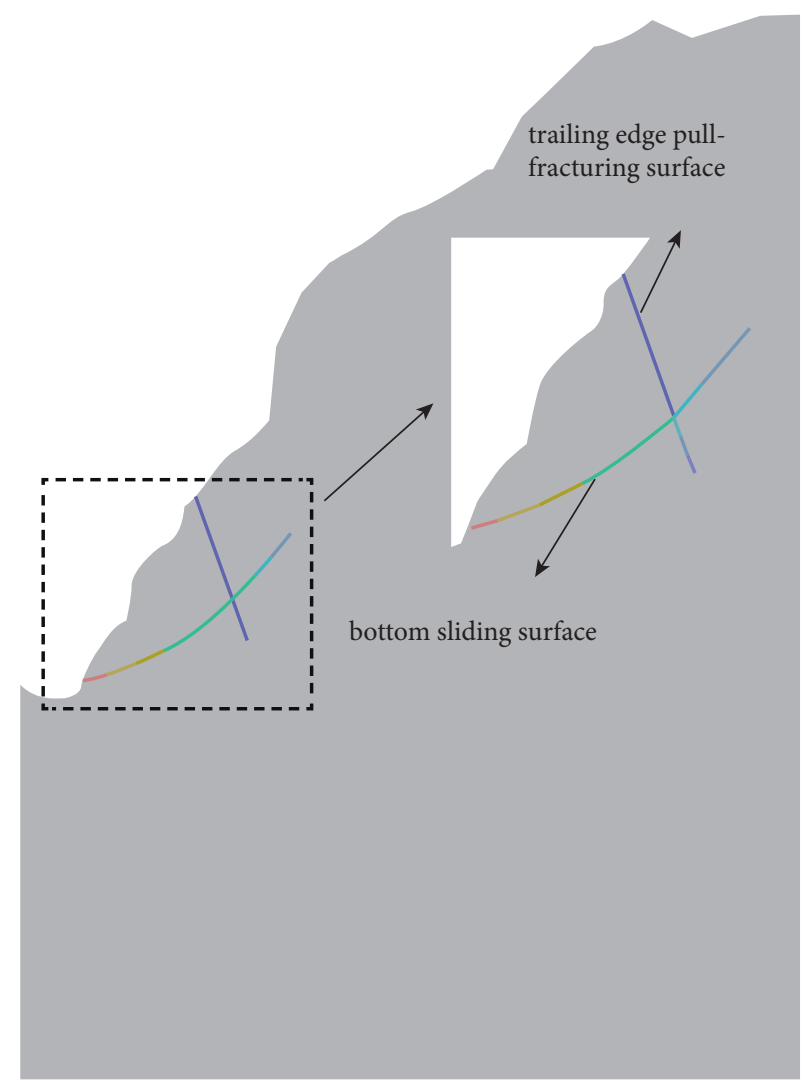

Figure 10: Distribution of plastic zone of the rock slope.

comprehensive consideration of various factors, the calculation result of the proportionality coefficient $k=1.2$ can be used as the comprehensive safety factor of the slope. When $k=1.2, K_{2}=1.867$, which meets the stability control standard 1.3. Therefore, the rock slope is relatively safe and stable. 
TABLE 6: Results of the safety factor of slope under different proportionality coefficient.

\begin{tabular}{lcccc}
\hline$k$ & $k_{c}$ & $k_{\varphi}$ & $K_{2}$ & Error (\%) \\
\hline 0.5 & 1.243 & 2.486 & 1.572 & -15.26 \\
0.6 & 1.381 & 2.302 & 1.675 & -9.70 \\
0.7 & 1.507 & 2.153 & 1.746 & -5.88 \\
0.8 & 1.629 & 2.036 & 1.799 & -3.02 \\
0.9 & 1.745 & 1.939 & 1.834 & -1.13 \\
1.0 & 1.855 & 1.855 & 1.855 & 0 \\
1.2 & 2.062 & 1.718 & 1.867 & 0.65 \\
1.4 & 2.272 & 1.623 & 1.868 & 0.70 \\
1.6 & 2.488 & 1.555 & 1.865 & 0.54 \\
1.8 & 2.712 & 1.507 & 1.863 & 0.43 \\
2.0 & 2.938 & 1.464 & 1.858 & 0.16 \\
\hline
\end{tabular}

Note. The calculation of the error is based on the calculation result of the traditional strength reduction method.

\section{Conclusion}

In this paper, the concrete application of double strength reduction factor method in the stability analysis of rock slopes was studied based on self-programmed program, and its applicability was verified through engineering examples. The following conclusions were drawn:

(1) The rock slope generally slides along the plastic zone formed by the weak interlayer zone, and the plastic zone obtained by the traditional strength reduction method and the double strength reduction coefficient method is basically the same.

(2) In the progressive failure process of rock slopes, the cohesion and internal friction angle play different roles, and their respective roles are mainly related to the inclination angle of the bottom sliding surface of the unstable block. If the inclination angle is less than $45^{\circ}$, the internal friction angle plays a greater role, and if the inclination angle is greater than or equal to $45^{\circ}$, the cohesion plays a greater role. Therefore, the double strength reduction factor method can be used to evaluate the stability of slopes more truly.

(3) Based on "Pan's principle," the internal force will automatically adjust to maximize the sliding resistance during the progressive failure of the slope. Therefore, when the inclination angle of the bottom sliding surface is less than $45^{\circ}$, the matching reduction principle of " $k_{c}>k_{\varphi}$ " can be adopted; when the inclination angle is more than $45^{\circ}$, the matching reduction principle of " $k_{c}<k_{\varphi}$ " can be adopted.

(4) The definitions of comprehensive safety factors $K_{1}$, $K_{2}$, and $K_{3}$ all have certain limitations, but $K_{2}$ is less affected by the value of $k_{c}$ and $k_{\varphi}$, so it is more suitable for the application of double strength reduction factor method in the stability analysis of rock slopes.

\section{Data Availability}

The programme data used to support the findings of this study are restricted by Hohai University in order to protect the patient privacy. Data are available from lujiaweihhu@ 126.com for researchers who meet the criteria for access to confidential data.

\section{Conflicts of Interest}

The authors declare that they have no conflicts of interest.

\section{Acknowledgments}

The project (U1765204) was supported by National Natural Science Foundation of China.

\section{References}

[1] Y.-r. Zheng and S.-y. Zhao, "Application of strength reduction FEM in soil and rock slope," Chinese Journal of Rock Mechanics and Engineering, vol. 23, no. 19, pp. 3381-3388, 2004, in Chinese.

[2] E. M. Dawson, W. H. Roth, and A. Drescher, "Slope stability analysis by strength reduction," Géotechnique, vol. 49, no. 6, pp. 835-840, 1999.

[3] Y. M. Cheng, T. Lansivaara, and W. B. Wei, "Two-dimensional slope stability analysis by limit equilibrium and strength reduction methods," Computers and Geotechnics, vol. 34, no. 3, pp. 137-150, 2007.

[4] W. B. Wei, Y. M. Cheng, and L. Li, “Three-dimensional slope failure analysis by the strength reduction and limit equilibrium methods," Computers and Geotechnics, vol. 36, no. 1-2, pp. 70-80, 2009.

[5] H. Zheng, G.-h. Sun, and D.-f. Liu, "A practical procedure for searching critical slip surfaces of slopes based on the strength reduction technique," Computers and Geotechnics, vol. 36, no. 1-2, pp. 1-5, 2009.

[6] E. Eberhardt, D. Stead, and J. S. Coggan, "Numerical analysis of initiation and progressive failure in natural rock slopes-the 1991 Randa rockslide," International Journal of Rock Mechanics and Mining Sciences, vol. 41, no. 1, pp. 69-87, 2004.

[7] D. Stead, E. Eberhardt, and J. S. Coggan, "Developments in the characterization of complex rock slope deformation and failure using numerical modelling techniques," Engineering Geology, vol. 83, no. 1-3, pp. 217-235, 2006.

[8] S.-y. Zhao, Y.-r. Zheng, and W.-d. Deng, "Stability analysis on jointed rock slope by strength reduction FEM," Chinese Journal of Rock Mechanics and Engineering, vol. 22, no. 2, pp. 254-260, 2003, in Chinese.

[9] F. Tang and Y.-r. Zheng, "Slope stability analysis based on two safety factors," Journal of Highway and Transportation Research and Development, vol. 25, no. 11, pp. 39-44, 2008.

[10] D. W. Taylor, Fundamentals of Soil Mechanics, John Wiley and Sons, New York, NY, USA, 1948.

[11] F. Tang and Y.-r. Zheng, "Analysis on safety reserve of slope with two strength reduction factor," Journal of Chongqing Jiaotong University (Natural Science), vol. 26, no. 4, pp. 95100, 2007, in Chinese.

[12] F. Tang, Y.-r. Zheng, and S.-y. Zhao, "Discussion on two safety factors for progressive failure of soil slope," Chinese Journal of Rock Mechanics and Engineering, vol. 26, no. 7, pp. 1402-1407, 2007, in Chinese.

[13] W. Yuan, X.-c. Li, W. Wang, B. Bai, Q.-Z. Wang, and X.-J. Chen, "Study on strength reduction method based on double reduction parameters," Rock and Soil Mechanics, vol. 37, no. 8, pp. 2222-2230, 2016, in Chinese. 
[14] Y.-p. Zhu, X.-y. Yang, R.-p. Yan et al., "Matching reduction mechanism of double reduction method," Journal of Lanzhou University of Technology, vol. 43, no. 2, pp. 121-126, 2017, in Chinese.

[15] X. Jiang, Z. Wang, L. Liu, and Z. Zhou, “The determination of reduction ratio factor in homogeneous soil-slope with finite element double strength reduction method," The Open Civil Engineering Journal, vol. 7, no. 1, pp. 205-209, 2013.

[16] H. Qi, L.-j. Zhang, C. Fang et al., "Dynamic stability analysis of slope based on dual reduction factor method," Water Resources and Power, vol. 36, no. 3, pp. 141-144, 2018, in Chinese.

[17] W. Yuan, B. Bai, X.-c. Li, and H.-b. Wang, "A strength reduction method based on double reduction parameters and its application," Journal of Central South University, vol. 20, no. 9, 2013.

[18] Y.-f. Chen, H. Lin, R.-h. Cao, and C. Zhang, "Slope stability analysis considering different contributions of shear strength parameters," International Journal of Geomechanics, vol. 21, no. 3, 2021.

[19] Y.-f. Chen, H. Lin, Y.-x. Wang, R.-h. Cao, C. Zhang, and Y. Zhao, "Modified double-reduction method considering strain softening and equivalent influence angle," KSCE Journal of Civil Engineering, vol. 24, no. 3, pp. 1976-3808, 2020.

[20] H.-b. Xue, F.-n. Dang, X.-t. Yin, C. Yang, and F. Yan, "Research on method of slope strength parameters non-proportional associated reduction," Chinese Journal of Rock Mechanics and Engineering, vol. 34, no. S2, pp. 4005-4012, 2015, in Chinese.

[21] F.-l. Zhang, Y.-h. Su, and L.-y. Guo, "Research on double reduction factors method based on soil softening law," Journal of Railway Science and Engineering, vol. 16, no. 9, pp. 22152222, 2019, in Chinese.

[22] A. Isakov and Y. Moryachkov, "Estimation of slope stability using two-parameter criterion of stability," International Journal of Geomechanics, vol. 14, no. 3, 2013.

[23] Y.-r. Zheng, S.-y. Zhao, and L.-y. Zhang, "Slope stability analysis by strength reduction FEM," Engineering Science, vol. 4, no. 10, pp. 57-61, 2002, in Chinese.

[24] Z.-y. Chen, "On Pan's principle of soil and rock stability analysis," Journal of Tsinghua Univer-sity (Science and Technology), vol. 38, no. 1, pp. 3-6, 1998, in Chinese. 